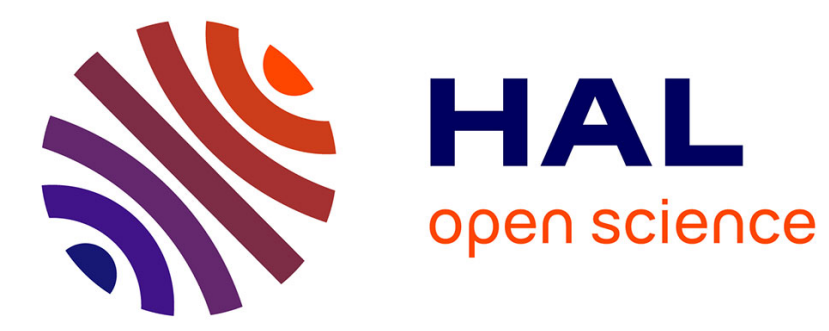

\title{
Le football saisi par les médias
}

Dominique Marchetti

\section{To cite this version:}

Dominique Marchetti. Le football saisi par les médias. Sociétés \& Représentations, 1998, Football \& Sociétés, 7, p. 309-331. 10.3917/sr.007.0309 . halshs-02379773

\section{HAL Id: halshs-02379773 https://shs.hal.science/halshs-02379773}

Submitted on 27 Nov 2019

HAL is a multi-disciplinary open access archive for the deposit and dissemination of scientific research documents, whether they are published or not. The documents may come from teaching and research institutions in France or abroad, or from public or private research centers.
L'archive ouverte pluridisciplinaire HAL, est destinée au dépôt et à la diffusion de documents scientifiques de niveau recherche, publiés ou non, émanant des établissements d'enseignement et de recherche français ou étrangers, des laboratoires publics ou privés. 


\section{LE FOOTBALL SAISI PAR LES MÉDIAS}

\section{Dominique Marchetti}

Éditions de la Sorbonne | «Sociétés \& Représentations 》

1998/2 Nº 7 | pages 309 à 331

ISSN 1262-2966

Article disponible en ligne à l'adresse :

https://www.cairn.info/revue-societes-et-representations-1998-2-page-309.htm

Distribution électronique Cairn.info pour Éditions de la Sorbonne.

(c) Éditions de la Sorbonne. Tous droits réservés pour tous pays.

La reproduction ou représentation de cet article, notamment par photocopie, n'est autorisée que dans les limites des conditions générales d'utilisation du site ou, le cas échéant, des conditions générales de la licence souscrite par votre établissement. Toute autre reproduction ou représentation, en tout ou partie, sous quelque forme et de quelque manière que ce soit, est interdite sauf accord préalable et écrit de l'éditeur, en dehors des cas prévus par la législation en vigueur en France. Il est précisé que son stockage dans une base de données est également interdit. 


\section{LE FOOTBALL SAISI PAR LES MÉDIAS}

$$
\text { pax Dominique Maxchetti }
$$

Ce programme de recherche s'inscrit dans un travail plus général sur les transformations du champ journalistique depuis le début des années Quatre-vingt et les relations que ce dernier entretient avec d'autres espaces sociaux. Ce projet vise notamment à comparer les changements qui ont affecté différents types de journalismes spécialisés. S'il existe des travaux français sur les journalistes politiques, sociaux, scientifiques et médicaux, les recherches sur le journalisme sportif sont rares. Seuls quelques essais journalistiques ou certaines monographies portant sur des rédactions ou des rubriques sportives apportent des éléments d'information. À partir de l'étude de la médiatisation de deux sports professionnels - le football et, de manière plus secondaire, le basket - il s'agit, d'une part, de prendre la mesure des transformations qui ont affecté le sous-champ du journalisme sportif ${ }^{1}$ depuis les années Quatre-vingt, du fait notamment de l'arrivée des télévisions privées, d'autre part, d'analyser comment les médias sont devenus un enjeu stratégique dans la production des «événements sportifs », ce qui a des effets sur le fonctionnement des espaces nationaux et internationaux des sports professionnels.

1. Si on étudie la presse spécialisée de diffusion nationale, il faut aussi et surtout analyser les rubriques sportives des médias nationaux d'information générale. La presse quotidienne régionale d'information générale a été écartée parce qu'elle mériterait, à elle seule, une enquête spécifique dans la mesure où le sport y occupe une place beaucoup plus grande que dans la presse quotidienne nationale. De plus, la hiérarchie des sports y varie suivant les régions. Sur ce sujet, cf. Alain Garrigou, «L'information sportive dans la presse régionale», in Albert Mabileau et André-Jean Tudesq, L'Information locale, Paris, Pedone, 1980, pp. 28-37; Pierre Thonat, Le Football dans les médias locaux, Rennes, mémoire de l'IEP de Rennes, 1998. Enfin, les radios, et notamment les stations nationales qui accordent une large place au sport, sont volontairement traitées de manière secondaire, car les archives, hormis dans le cas de Radio France, n'existent pas ou sont d'un accès difficile. 


\section{«L'EXPOSITION MÉDIATIQUE» D'UN SPORT}

Quelle est la place occupée par le football et le basket dans la médiatisation du sport en France? Le football est non seulement le sport qui compte le plus de licenciés (2,05 millions en 1997) mais il est aussi celui qui est le plus médiatisé. Ainsi, à la télévision, représente-t-il l'essentiel des retransmissions en direct à des heures de grande écoute sur les chaînes généralistes en clair. Ce poids s'est renforcé avec le développement du football professionnel et l'arrivée des chaînes privées. Le nombre de matchs retransmis s'est multiplié avec les contrats passés par Canalt. Ainsi, le football représentait-il, en 1997, 402 heures et 17 minutes si on totalise les temps d'antenne de TF1 (152 h), France 2 ( 1 h 37), France 3 ( 48 h 40) et surtout de Canal+ $(200 \mathrm{~h})^{2}$. Le volume des retransmissions et des émissions de football sur les chaînes hertziennes a été multiplié par deux en six ans : 483 heures en 1995 contre 233 heures en 19893 . La forte exposition médiatique de ce sport se mesure également à travers la place qu'il occupe dans les quotidiens nationaux et régionaux. Par exemple, à L'Équipe, le football occupait, en 1995, 25 à $27 \%$ de la surface rédactionnelle et, selon les enquêtes, $88 \%$ des lecteurs déclaraient lire cette rubrique 4 . Enfin, les plus gros tirages de la presse sportive, si l'on excepte L'Équipe, sont réalisés par des titres qui traitent exclusivement du football : France Football - qui a augmenté sensiblement sa diffusion depuis le début des années Quatre-vingt (une moyenne de 230554 exemplaires en 1996 contre 144245 en 1981) ${ }^{5}$, développant deux éditions le mardi (199175 exemplaires en 1997) et le vendredi (90918) - et le mensuel Onze Mondial (161661). Le basket, qui est le cinquième sport en nombre de licenciés (437974 en 1997), occupe une position médiane entre les sports professionnels ou semi-professionnels (athlétisme, judo, escrime) qui ont peu d'exposition médiatique, hormis lors des Jeux Olympiques, et les sports dominants. En 1997, il arrive en sixième position pour l'exposition à la télévision avec 100 heures.

L'emploi de l'expression «exposition médiatique» demeure insatisfaisant. Si l'offre des sports et des événements couverts - c'est vrai pour le football et le basket -, notamment par les télévisions depuis le milieu des années Quatre-vingt, a glo-

2. Source : Conseil Supérieur de l'Audiovisuel (CSA).

3. Conseil Supérieur de l'Audiovisuel, Sport et télévision (1991-1996). Bilan de six années de régulation, Paris, mars 1997, p. 113.

4. Ce chiffre est extrait d'un article du Monde daté du 24 fév. 1996. Par ailleurs, selon l'enquête de Jérémie Arbona (La Rédaction du journal L'Équipe, Paris, mémoire de maîtrise d'information et communication de l'Institut français de presse, Université Paris II, septembre 1997) sur 77 éditions de L'Équipe du premier semestre 1997, 39 «unes» ont été consacrées majoritairement au football.

5. Ces chiffres concernent la diffusion totale France (source : Diffusion contrôle). 
balement augmenté, cette évolution cache au moins deux tendances. D'une part, des médias de plus en plus spécialisés se sont développés, comme le montre la création de chaînes de radio (Sport O'FM) ou de télévision (Eurosport France, AB Sport, L'Équipe TV, Infosport), partiellement ou uniquement consacrées au sport, mais surtout l'augmentation du nombre de magazines : les exemples du football et surtout du basket (américain essentiellement) en témoignent. D'autre part, au sein de l'espace des médias nationaux grand public, l'intensification de la concurrence commerciale par la création de chaînes privées a eu deux effets majeurs. Si depuis la moitié des années Quatre-vingt, le football est l'un des rares sports à faire l'objet de retransmissions régulières sur $\mathrm{TF} 1$, et quelquefois sur les chaînes du service public, il est de moins en moins visible en clair $(50 \%)^{6}$. Une grande part des retransmissions de matchs sont et seront de plus en plus à «double péage» en raison du développement des paiements à la séance ou pay per view, dont l'accès n'est possible qu'aux abonnés du câble ou du satellite 7 . La concurrence commerciale a conduit en second lieu à accroître le degré d'exposition médiatique des sports professionnels dominants. Les sports qui sont censés faire moins d'audience ont disparu, les responsables des chaînes tendant à se concentrer sur quelques sports comme la Formule 1 et le football, voire, pour certains événements, sur le cyclisme, la boxe ou le rugby. C'est surtout à propos de ces sports que la concurrence pour l'achat des droits de retransmission est exacerbée. C'est ainsi que le basket est, sous ce rapport, dans une situation comparable à celle du tennis (si l'on excepte Roland Garros, le tournoi de Bercy ou la coupe Davis), c'est-à-dire qu'il a disparu des programmes des chaînes généralistes en clair : il est devenu un «sport à péage», qui n'est visible qu'à condition d'être abonné au câble, au satellite ou à Canal+.

À la différence du football, le basket professionnel français, qui s'oppose, par ailleurs, sous beaucoup de rapports à son homologue américain plus «médiatique», présente, en effet, certaines propriétés qui le rendent peu intéressant aux yeux des journalistes. Par exemple, c'est un sport jugé «trop technique» dont les règles sont «compliquées». Il n'y a pas pour l'instant de grands champions aux caractéristiques «médiatiques», c'est-à-dire susceptibles de dépasser le seul public des

\section{L'Équipe, 20-21 déc. 1997.}

7. Ce système, très développé aux États-Unis mais qui existe depuis les années Quatre-vingt-dix en France, permet de regarder un match «à la carte», moyennant un paiement à l'année ou à chaque compétition organisée. Ainsi, les matchs du championnat de France de division 1 qui ne sont pas diffusés par Canal+, sont visibles sur Kiosque, détenu par cette même chaîne. Le service Multivision, disponible sur certains réseaux câblés et sur le bouquet satellite TPS, permet aussi d' "acheter» des rencontres de la plus prestigieuse compétition européenne, la ligue des champions. 
«connaisseurs». Les mauvais résultats de l'équipe de France - qui n'est pas parvenue à se qualifier pour les Jeux Olympiques depuis ceux de Los Angeles - et des clubs à l'échelon européen contribuent également à expliquer la faible place occupée par le basket français dans les médias nationaux ${ }^{8}$.

Pour comprendre l'état actuel de la médiatisation du football et du basket, il faut mettre en relation la constitution et le développement des espaces nationaux de ces deux sports professionnels et les transformations du champ journalistique9. En effet, contrairement à une idée souvent répandue, la visibilité médiatique d'un sport ne dépend pas uniquement de la variation du nombre officiel de ses pratiquants, de ses licenciés ou de son audience telle qu'elle est mesurée à travers les sondages. Il suffit d'introduire des éléments de comparaison à l'échelon international pour montrer le poids des spécificités nationales. Pour définir ces dernières il convient d'analyser la place d'un sport considéré dans l'espace national des sports, saisir les groupes sociaux différents que ce spectacle sportif mobilise, mettre au jour les différences des systèmes politiques et économiques. Par exemple, le basket est beaucoup plus développé et médiatisé en Italie et en Espagne, où il figure derrière le football, qu'en France. La situation est inversée pour le rugby. Comment expliquer également la médiatisation de certains sports universitaires (le basket et le football américain notamment) aux États-Unis alors qu'ils sont souvent ignorés en France ? ${ }^{10}$. Celle-ci doit être étudiée à l'échelle globale du journalisme, c'est-à-dire dans ses rapports avec les transformations qu'a connues le champ journalistique depuis les années Quatre-vingt. Ensuite, il faut distinguer les différents sous-secteurs de concurrence qui obéissent en partie à des logiques propres, qu'il s'agisse des types de presse (presse spécialisée, presse quotidienne, news magazines, radio, télé) et des rédactions qui constituent autant de sous-espaces de concurrence. À ces différents niveaux d'analyse, il faut en ajouter un dernier, le sous-secteur du journalisme sportif sur lequel portera l'essentiel de cette esquisse.

\footnotetext{
8. Ces reproches sont invoqués par de nombreux rédacteurs en chef de rubriques sportives de grands médias : cf. «Le basket est absent des médias », Basket Hebdo, 1er avril 1998, pp. 8-9. 9. Sur cette notion, $c f$. Jean-Michel Faure et Charles Suaud, «Les enjeux du football», Actes de la recherche en sciences sociales, ${ }^{\circ} 103,1994$, pp. 3-6.

10. Sur ces questions touchant à la genèse de la professionnalisation des sports, on peut s'appuyer sur les travaux de Jean-Michel Faure et Charles Suaud portant sur la sociologie du football et sur ceux de Sylvain Robert dans le cas du basket. Pour analyser la médiatisation de ces deux sports, on combinera donc les acquis de ces travaux avec une étude spécifique sur la médiatisation du football et du basket. Jean-Michel Faure et Charles Suaud, «Le professionnalisme inachevé. Deux états du champ du football professionnel en France, 1963-1993», Actes de la recherche en sciences sociales, $\mathrm{n}^{\circ} 103,1994$, pp. 7-25; Sylvain Robert, Une structuration inachevée. Processus à l'oeuvre dans la formation d'un sport-basket de haut niveau en France (formes et enjeux 1920-1997), thèse de sociologie, Université de Nantes, 1997.
} 


\section{LE SOUS-CHAMP DU JOURNALISME SPORTIF}

Bien que les journalistes sportifs soient relativement nombreux ${ }^{11}$ et que les rubriques sportives se soient considérablement développées ${ }^{12}$, le journalisme sportif occupe une position notoirement dominée dans l'espace journalistique, dont l'évidence s'exprime souvent dans les discours des journalistes sportifs euxmêmes. Ainsi, un document de l'Union syndicale des journalistes sportifs de France montre-t-il que «le journaliste sportif» est souvent perçu par ses confrères comme un «professionnel d'un type un peu particulier, un peu marginal, parfois dédaigné comme la matière qu'il traite $»^{13}$. Dans les caricatures du milieu, il est décrit comme un «animateur», un supporter, voire un «frimeur»: «l'image du journaliste sportif, blouson de cuir sur muscles apparents et bronzage permanent, mi-grand frère, mi-prof de gym, a de quoi séduire les fans de L'Équipe et les mordus des retransmissions télévisées», explique ainsi, de manière caricaturale, l'auteur d'un manuel sur le journalisme ${ }^{14}$. Comme l'écrivent Charles Suaud et Jean-Michel Faure, cette position dominée induit des pratiques professionnelles qui se caractérisent par une «grande proximité avec le terrain, que l'on observe dans le ton des reporters, dans certaines facilités de langage socialement (et professionnellement) acceptées, ou encore dans l'adoption d'un style obligé fait de passion, de camaraderie populaire et d'exaltation de valeurs consensuelles ${ }^{15}$. Alain Garrigou a bien décrit cette «rhétorique de l'information sportive» qui souligne l'héroïsme ou emprunte des termes au «monde du travail», qu'ils soient positifs («souffrance», «rendement») ou négatifs («laborieux $»)^{16}$.

En France, le journalisme sportif offre la même structure que le champ journalistique global :

- un pôle «commercial», incarné par le traitement du sport - constitué essentiellement de résultats et de comptes rendus - dans les quotidiens populaires nationaux ou régionaux ou encore les grandes chaînes de télévision et de radio;

11. À la fin des années Quatre-vingt, sur les 23000 journalistes en activité, 3000 environ traitaient le sport, dont deux tiers en permanence : $c f$. Jacques Marchand, La Presse sportive, Paris, éd. du CFPJ, 1989, p. 47.

12. À titre indicatif, on peut signaler que, selon le directeur du service des sports de l'Agence France Presse, le sport représentait en moyenne, à la fin des années Quatre-vingt, 25 à $30 \%$ de la copie diffusée en six langues par l'agence.

13. Extrait du préambule de L'Essai sur les responsabilités des journalistes dans les déviations du sport (Union des journalistes sportifs français, 1975), cité par Gérard Derèze, «Le petit monde des journalistes sportifs de télévision. Représentations de rôles en Belgique francophone», Réseaux, $\mathrm{n}^{\circ}$ 57, 1993, p. 52.

14. Christian Sauvage, Journaliste, une passion, des métiers, Paris, éd. du CFPJ, 1988.

15. Jean-Michel Faure et Charles Suaud, «Les enjeux du football», loc. cit., p. 6.

16. Alain Garrigou, «L'information sportive dans la presse régionale», loc. cit., p. 34. 
- un pôle plus «intellectuel» où l'on retrouve les news magazines et les quotidiens dits «de qualité» (Le Monde, Libération, Le Figaro notamment) qui considèrent davantage d'autres aspects des activités sportives : économiques, politiques, médiatiques. Les premiers éléments de l'enquête permettent d'avancer deux hypothèses. Comme c'est le cas dans d'autres sous-espaces spécialisés du journalisme, le pôle «commercial» s'est renforcé, notamment avec l'arrivée des chaînes de télévision privées. Par ailleurs, le mode de traitement de l'information sportive tend, semble-t-il, à devenir de plus en plus homogène ${ }^{17}$, l'opposition entre les deux pôles devenant de moins en moins affirmée.

La structure du journalisme sportif s'articule aussi autour d'une deuxième opposition. Il y a, d'une part, un pôle «généraliste-grand public» - représenté notamment par les quotidiens et les chaînes de télé et de radio omnibus pour lesquels la médiatisation du sport se réduit à quelques sports professionnels dominants : le football, la Formule 1, le rugby et le cyclisme - et, d'autre part, un pôle plus spécialisé au sein duquel le degré de spécialisation varie fortement : dans la presse écrite, s'opposent, d'un côté, le quotidien L'Équipe, qui traite différents sports mais donne la priorité à quelques-uns (football, rugby, tennis, cyclisme, automobile) et, de l'autre, les magazines ou les médias spécialisés dont la diffusion est plus restreinte et qui se sont très fortement développés dans les années Quatre-vingt et Quatre-vingt-dix. L'opposition «généraliste»/《 spécialiste» s'observe bien évidemment dans les différents supports. Par exemple, dans le cas de la télévision, les chaînes généralistes qui ne s'intéressent qu'à un petit nombre de sports ne lẹur appliquent pas le même traitement que les chaînes spécialisées comme Eurosport France, la situation étant probablement comparable à l'étranger avec les chaînes sportives : DSF en Allemagne, Sky Sport en Angleterre ou ESPN aux États-Unis.

Enfin, une dernière opposition existe dans le journalisme sportif en France, entre la presse écrite et les médias audiovisuels, et surtout la télévision. Les journalistes sportifs de la presse écrite nationale peuvent être prédominants sous le rapport de la légitimité professionnelle, c'est-à-dire qu'ils sont souvent considérés par leurs confrères comme plus prestigieux que ceux de la télévision, parfois traités avec mépris comme des «marchands de soupe», certains allant jusqu'à leur refuser le titre de journaliste. À l'inverse, la légitimité externe des journalistes de télévision est beaucoup plus forte auprès du public - qui les connaît et les reconnaît - et des

17. Par exemple, le traitement du sport dans Le Monde et Libération a eu des effets sur celui de L'Équipe et inversement. Les «transferts» de journalistes d'un média à l'autre (ou du Parisien au Monde par exemple) sont des indicateurs de l'homogénéisation du traitement de l'information sportive. 
sportifs. Même si cela varie suivant les médias, la «visibilité» télévisuelle est très appréciée pour différentes raisons, dont, entre autres, ses effets de publicité pour les sponsors. Cette hiérarchie est, par exemple, très visible les soirs de matchs de football rassemblant de nombreux journalistes ou lors de grandes compétitions internationales. Les journalistes de télévision peuvent souvent interviewer en priorité les sportifs tandis que leurs confrères de la presse écrite doivent se contenter d'une conférence de presse. Cette hiérarchie ne va pas sans provoquer des tensions qui se traduisent par la critique de l'«arrogance» des journalistes des grandes chaînes de télévision ( «Ils se croient tout permis», «Ce sont les rois», entend-on parfois).

Au sein de la structure du sous-espace du journalisme sportif, le poids de chacun des supports dans la production de l'information sportive pour le grand public est très inégal. Par rapport au champ journalistique, la principale spécificité de ce sous-champ est la position dominante occupée par L'Équipe, créé le 28 février 1946. Elle est d'autant plus forte aujourd'hui que son concurrent Le Sport (1987-1988) a disparu. L'Équipe est le seul quotidien sportif français, même s'il se retrouve objectivement en concurrence avec les quotidiens régionaux, et notamment Le Parisien, qui accordent une large place à l'actualité sportive. Au-delà de cette situation de monopole, c'est son histoire et sa diffusion, notamment dans les milieux sportifs où il est très lu, qui fondent son prestige à tel point qu'il est parfois appelé «le journal officiel du sport français». Il occupe mutatis mutandis une position homologue à celle du Monde dans le journalisme politique. L'impact médiatique de la "polémique» entre Aimé Jacquet et la direction du quotidien sportif, avant, pendant et après l'édition 1998 de la Coupe du Monde de football, a été assez révélateur sous ce rapport. On peut rendre compte de cette position en se fondant sur plusieurs indicateurs. Ainsi, ce quotidien constitue-t-il à des degrés divers la lecture quotidienne des journalistes sportifs, qui reprennent souvent ses informations exclusives et s'informent à travers lui. Sa lecture fait partie de l'apprentissage professionnel du jeune journaliste sportif. Les premiers éléments de notre enquête montrent également qu'être journaliste à L'Équipe, c'est occuper une des positions les plus prestigieuses (et donc les plus convoitées) dans ce sousespace de production journalistique. Au prestige interne s'ajoute le prestige externe du quotidien auprès des sportifs, y compris à l'étranger.

Les chaînes de télévision, c'est-à-dire essentiellement Canal+ et TF1 quand il s'agit de football, occupent également une position dominante dans la médiatisation du football. Par exemple, celle-ci peut être analysée à travers les effets des retransmissions des chaînes de télévision sur la presse écrite. On pourrait montrer combien la hiérarchie du sport à la télévision pèse sur celle de la presse écrite qui 
privilégie très souvent les événements sportifs consacrés par la télévision. La «visibilité» d'un sport à la télévision pèse très directement dans les luttes internes aux rédactions sportives des autres médias. L'audimat, ou plus précisément la lecture qu'en font les journalistes, est une arme dans les débats, en conférences de rédaction, sur la surface rédactionnelle à accorder à tel ou tel sport ou événement. Ainsi, par exemple, les remarques qu'on entend fréquemment à propos du basket désigné comme «un sport qui n'intéresse pas grand monde» s'appuient-elles largement sur la faible audience du basket français à la télévision, qui a été soulignée par les responsables des sports de Canal+ et de France Télévision pour justifier le désengagement total ou partiel de leurs chaînes. C'est particulièrement vrai dans le cas de L'Équipe qui réalise ses meilleures ventes lors des événements télévisés : les finales des grandes compétitions internationales de football (Coupe du Monde, Ligue des Champions), les grands prix de Formule 1, les Jeux Olympiques, le Tour de France, Roland Garros. Le développement de rubriques spécifiques sur le sport à la télévision dans la presse écrite, et plus encore la place prise par le sport télévisé dans les rubriques sportives ou télévisuelles sont un indice de la position forte qu'occupe aujourd'hui la télévision dans la production de l'information sportive.

L'analyse des positions occupées par les différents médias dans le journalisme sportif fait également apparaître le poids spécifique de l'Agence France Presse. Non seulement elle fournit des informations propres qui constituent une matière importante pour les autres médias, mais elle a également un pouvoir de consécration interne très important. À l'instar d'autres secteurs comme la médecine ${ }^{18}$, la «reprise» d'une information de tel ou tel média par l'AFP lui donne un crédit journalistique dans la mesure où elle l'«officialise».

Enfin, le sous-champ du journalisme sportif se structure non seulement suivant les différents types de médias, mais aussi selon les sports considérés. Cette hiérarchie apparaît dans les luttes quotidiennes pour la répartition rédactionnelle entre les différents rubricards au sein des rédactions sportives. Une enquête permettrait de vérifier que la hiérarchie des journalistes sportifs reproduit bien la hiérarchie de l'audience des sports : s'opposeraient ainsi les journalistes qui traitent des sports les plus populaires, comme le football - ils sont souvent considérés comme plus prestigieux (on parle parfois de l'«aristocratie» au journal L'Équipe) -, et les journalistes qui couvrent des disciplines dont l'audience est plus restreinte.

18. Dominique Marchetti, Contribution à une sociologie des transformations du champ journalistique dans les années Quatre-vingt et Quatre-vingt-dix. À propos d' 'événements sida et du "scandale du sang contaminé", Paris, thèse de sociologie, École des Hautes Études en Sciences Sociales, 1997. 


\section{LES TRANSFORMATIONS DU RECRUTEMENT DES JOURNALISTES}

Analyser les transformations du journalisme sportif, c'est aussi saisir les mutations de la population des journalistes sportifs travaillant dans les médias nationaux. Depuis le début des années Quatre-vingt, l'offre de travail s'est considérablement accrue avec le développement des médias spécialisés (par exemple Canal+ employait cinq journalistes au service des sports en 1984, ils étaient soixante-cinq en 1996) et les départs en retraite des nombreux journalistes sportifs qui ont commencé leur carrière professionnelle dans les années Quarante et Cinquante. Tout laisse à penser que les transformations morphologiques de la population des journalistes sportifs suivent, dans leur grandes lignes, celles qui ont affecté l'ensemble des journalistes professionnels dans les années Quatre-vingt et Quatrevingt-dix ${ }^{19}$ : augmentation des effectifs, accroissement du niveau de diplôme, rajeunissement et féminisation. On peut également faire l'hypothèse que la part des diplômés des écoles de journalisme, parmi les journalistes sportifs entrés dans les grands médias nationaux à partir du début des années Quatre-vingt, est en forte augmentation, confirmant ainsi une tendance qui est manifeste dans l'ensemble de ces médias. Les jeunes entrants issus de ces formations sont de plus en plus nombreux et plusieurs anciens élèves de ces écoles, comme Noël Couedel à L'Équipe puis au Parisien, Gérard Ernault, Jean-François Renault à L'Équipe, Charles Bietry, Gérard Marcout à Canal+, occupent ou occupaient des postes de responsables. Si la cooptation d'anciens sportifs, qui est une pratique ancienne (les athlètes Marcel Hansenne et Michel Clare à L'Équipe, le rugbyman Pierre Albaladejo ou encore le footballeur Jean-Michel Larqué à la télévision), et le recrutement de journalistes formés sur le tas, tout particulièrement dans la presse quotidienne régionale, subsistent dans les grands médias nationaux, le passage par une école fait aujourd'hui figure de «voie royale ${ }^{20}$. Plus généralement, le niveau d'études des journalistes sportifs, notamment dans les médias les plus prestigieux (les chaînes nationales de télévision, de radio et L'Équipe par exemple), paraît de plus en plus élevé.

Au-delà de ces tendances qui affectent l'ensemble de la population des journalistes professionnels, une enquête pourrait permettre de dégager les spécificités des journalistes sportifs. On peut cependant avancer plusieurs tendances qui semblent se dégager d'un travail liminaire. La première est que l'origine sociale des journalistes sportifs reste toujours plus «populaire» que celle de la moyenne des

19. Valérie Devillard, Marie-Françoise Lafosse, Jean-Pierre Maruhenda, Rémy Rieffel, Les Journalistes français en 1990, radiographie d'une profession, SJTI-La Documentation française, 1991.

20. Dominique Marchetti, Contribution à une sociologie..., op. cit. 
journalistes. Une étude réalisée à l'Institut français de presse ${ }^{21}$, portant sur un petit échantillon de journalistes qui exercent dans des rubriques sportives, paraît confirmer ce constat très ancien : $42 \%$ étaient des fils d'employés ou d'ouvriers contre $10 \%$ pour l'ensemble des journalistes titulaires de la carte de presse. Bien évidemment, il faudrait établir les variations de l'origine sociale suivant les supports considérés - par exemple, à L'Équipe, sur les 28 journalistes interrogés lors d'une enquête ${ }^{22}, 16$ avaient un père et/ou une mère cadre supérieur - et les sports traités. En outre, les producteurs de l'information sportive sont très majoritairement des hommes, tout comme les lecteurs des pages consacrées au sport ou de la presse sportive en général (par exemple, 85 \% des lecteurs dans le cas de L'Équipe). Ainsi, sur les 1800 adhérents que comptait l'Union syndicale des journalistes sportifs de France (USJSF) ${ }^{23}$ en 1988, 38 étaient des femmes. Sur 82 journalistes de L'Équipe en 1996, 7 sont des femmes. La troisième spécificité de cette population est liée, semble-t-il, à sa faible mobilité interne, les professionnels employant l'expression de «ghetto" pour évoquer la rubrique sportive. Cependant, certains cas de mobilité professionnelle - par exemple, un ancien journaliste sportif du quotidien Le Sport, qui a intégré la rubrique sport du Monde avant de devenir grand reporter dans ce quotidien, ou des reporters sportifs exerçant dans des médias audiovisuels qui sont devenus présentateurs de journaux - mériteraient d'être analysés pour mieux comprendre ces «exceptions» et peut-être nuancer cette hypothèse.

\section{LE POIDS DES LOGIQUES COMMERCIALES}

Un troisième axe de recherche vise à saisir les transformations des contraintes de production qui pèsent sur l'activité des journalistes sportifs. Il faudrait, par exemple, mesurer les effets très importants de l'introduction des nouvelles techniques, à la télévision comme dans la presse écrite, sur la médiatisation du sport : caméras plus légères, transmission par satellites, téléphones et ordinateurs portables. En effet, le journalisme sportif, à la radio ${ }^{24}$ et à la télévision notamment, a historiquement permis l'introduction des nouvelles techniques de transmission en direct qui ont été ensuite généralisées. Mais on insiste ici sur une de ces contraintes,

21. Olivier Escarmena, Les Journalistes sportifs : profils et caractéristiques d'un journalisme spécialisé, Paris, mémoire de maîtrise de l'Institut français de presse, Université Paris II, sept. 1993.

22. Jérémie Arbona, La Rédaction du journal L'Équipe, op. cit.

23. Créée le 18 janvier 1958, l'Union syndicale des journalistes sportifs de France est la principale association de journalistes sportifs.

24. Sur la naissance du radio-reportage, $c f$. Cécile Méadel, «De l'épreuve et de la relation. La genèse du radio-reportage », Politix, $\mathrm{n}^{\circ} 19,1992$, pp. 87-101. 
la contrainte économique. Pour rendre compte des mécanismes par lesquels elle s'exerce, on se propose d'analyser l'intensification de la concurrence commerciale que se livrent aujourd'hui les différents supports de presse. C'est particulièrement vrai pour les chaînes de télévisions où la concurrence se règle, à la différence d'autres sous-espaces spécialisés, souvent moins à travers la diffusion de telle ou telle information exclusive que par l'achat des droits de retransmission des grands événements sportifs, ceux-ci ayant considérablement augmenté avec l'arrivée des chaines de télévision privées ${ }^{25}$. À titre indicatif, le prix d'un match de coupe d'Europe diffusé en 1982 sur TF1, qui était alors une chaîne publique, a été multiplié par cinquante, six ans plus tard, après la privatisation de TF1 : de 1982 à 1988, le coût est passé de 150000 francs à 7,5 millions de francs ${ }^{26}$. Avec le développement des réseaux câblés et des bouquets satellites, la concurrence pour l'achat des matchs est exacerbée dans le cas des sports qui attirent un large public, tout particulièrement pour le football qui est, avec le cinéma, un des principaux produits d'appel pour les programmes proposés par les deux principaux opérateurs français : Vivendi (ex-Générale des eaux), qui est présent dans Canal+ et NC Numéricâble, et La Lyonnaise des eaux, qui possède des parts dans le bouquet satellite TPS et Lyonnaise câble. Les tensions récentes à propos des droits de retransmission des matchs du championnat de France de division 1 (finalement détenus par Canal+), des coupes nationales et des matchs des coupes d'Europe (où il y a eu un partage), notamment de la plus prestigieuse d'entre elles, la ligue des champions, ont confirmé l'importance de l'enjeu commercial que représente désormais le football, surtout pour le lancement ou le développement de nouveaux bouquets de chaînes numériques.

Mais l'intensification de cette concurrence commerciale doit aujourd'hui être saisie plus largement à l'échelon international puisqu'elle implique des groupes de communication qui possèdent des chaînes dans de nombreux pays - le football n'étant qu'un des secteurs où se joue la concurrence pour les parts de marché - et qui sont incarnés par des patrons emblématiques comme l'Italien Silvio Berlusconi, l'Allemand Léo Kirch, l'Australien (naturalisé américain) Rupert Murdoch et le Français Pierre Lescure. L'un des objets de cette recherche serait de faire la genèse de cette concurrence pour les droits et de voir plus largement quelles formes prend cette concurrence commerciale entre les chaînes de télévision.

25. Conseil Supérieur de l'Audiovisuel, Le Sport et la Télévision. Analyse, avis et propositions, Paris, juill. 1991.

26. Exemple cité par Éric Maitrot, Sport et Télé. Les liaisons secrètes, Paris, Flammarion, 1995, p. 45. 
Pour saisir le poids des logiques économiques, non plus à la télévision mais dans la presse écrite, on s'intéressera plus particulièrement à l'importance prise par les études de lectorat, qui explique en partie que le sport soit aujourd'hui une des rubriques les plus développées dans l'ensemble de la presse nationale, qu'il s'agisse bien évidemment de la presse populaire ${ }^{27}$ mais aussi de la presse dite «de qualité ». Le journal L'Équipe en fournit un bon exemple puisqu'il a développé, à partir de 1991, avec l'institut BVA, des études de plus en plus fines sur ses lecteurs, étoffant également son service marketing. Par rapport aux autres quotidiens nationaux, la part des ventes dans les recettes est beaucoup plus élevée : $80 \%$ contre $60 \%$ en moyenne pour ses confrères. Le renouvellement progressif, à partir du départ de Jacques Goddet en 1984 de l'équipe dirigeante «administrative», composée pour partie de personnes ayant travaillé dans la publicité, n'a probablement pas été sans effet sur le fonctionnement du quotidien. L'Équipe s'est progressivement transformé à partir de la deuxième moitié des années Quatre-vingt : ce «journal de spécialistes", pour reprendre une formule de son directeur actuel, est devenu plus "grand public», touchant des lecteurs qui se disent, selon les enquêtes, de plus en plus «zappeurs», c'est-à-dire passant d'une rubrique à l'autre. Il est, avec Le Parisien, le principal succès économique de la presse quotidienne en France depuis le milieu des années Quatre-vingt : sa diffusion totale payée a augmenté de $70 \%$ entre 1987 et 1997 (386294 exemplaires contre 222544).

Enfin, l'une des manières de détailler le poids de ces logiques commerciales est l'analyse de la montée de la précarité - ce phénomène concerne à des degrés divers l'ensemble de l'activité journalistique - chez les journalistes sportifs qui travaillent notamment pour la presse magazine, laquelle repose sur de petites structures rédactionnelles étoffées par de nombreux pigistes. Ce n'est pas un hasard si c'est un des axes de la «politique» de l'Union syndicale des journalistes sportifs de France.

Mais cette description du renforcement des logiques commerciales dans les grands médias nationaux n'a d'intérêt que si elle est complétée par une étude de ses effets sur l'activité journalistique. L'attention sera portée sur certains métiers du journalisme en prenant l'exemple du poste de responsable des sports, notamment au sein des chaînes de télévision de diffusion nationale. Celui-ci a considérablement évolué puisqu'il demande aujourd'hui plus que des compétences journalistiques ${ }^{28}$, les titulaires de ces postes ayant d'ailleurs souvent cessé de

27. Cette situation est encore plus flagrante en Angleterre : $c f$. Jean K. Chalaby, The Invention of Journalism, London-New York, MacMillan Press-St. Martin's Press, 1998, p. 92.

28. Cette tendance est plus ancienne aux États-Unis. Un responsable du service des sports d'un journal américain expliquait en 1980 : «With higher staff salaries and much larger travel budgets, contemporary sports editors manage a sizable investment of their newspapers. It is a critical job demanding extremely well-qualified people». Cité par Bruce Garrison avec Mark Sabljak, Sports Reporting, Ames, Iowa State University Press, 1993 (second ed.), pp. 313-314. 
présenter des émissions ou de commenter des compétitions. La gestion de personnels et de budgets de plus en plus importants - par exemple, le budget «sports» de Canal+, qui était de 32 millions de francs en 1985, est vingt-quatre fois plus important dix ans plus tard : 782 millions de francs en $1995^{29}$-, les discussions avec la direction des programmes, et surtout les négociations pour l'achat des droits de retransmission, forment aujourd'hui une partie essentielle de l'activité d'un directeur des sports d'une grande chaîne de télévision.

Les impératifs commerciaux ont surtout des effets sur le traitement de l'information. On peut avancer quelques pistes de recherches. L'évolution de L'Équipe pourrait fournir un premier cas révélateur. Comme l'a montré une enquête ${ }^{30}$ qui pourrait être développée à l'aide d'outils statistiques, la nécessité d'attirer le plus grand nombre de lecteurs se traduit par des manières spécifiques de traiter le sport : l'importance accordée à l' «événement» ou à l' «actualité chaude», la modification, en 1987, de la «une» - l'adoption de la couleur, un titre accrocheur et peu de mots, la priorité donnée aux sports et aux équipes (1'Olympique de Marseille et le PSG) qui attirent le public, le choix de «parler des stars ${ }^{31}$ des sports majeurs, la multiplication des photos, les reportages sur la vie des sportifs ou sur les sujets plus «loisirs» et le recours fréquent aux questions-réponses.

Les effets de la logique économique sont probablement encore plus manifestes dans le traitement des événements sportifs à la télévision. Ainsi, la nécessité de rentabiliser les investissements consentis pour certaines épreuves (avions, hélicoptères, motos, personnels sur le terrain) explique que la durée des retransmissions soit plus longue qu'auparavant. Les contraintes économiques se traduisent même dans les commentaires : comme le dit, avec un air amusé, un ancien responsable des sports d'une chaîne publique, «on ne peut plus dire que ça ne vaut pas un clou, vu le prix que ça coûte» ${ }^{32}$. En effet, l'autonomie des journalistes sportifs est de plus en plus faible, du fait qu'ils relatent très souvent un spectacle dont leur chaîne est l'un des coproducteurs. Ces contraintes sont bien décrites, dans un ouvrage récent ${ }^{33}$, par certains journalistes de France 2 qui affirment crûment qu'ils

29. Chiffres cités par Vincent Celati, Le Service des sports de Canal+, Paris, mémoire pour le diplôme de l'Institut Français de Presse, Université Paris II, 1996-1997.

30. Jérémie Arbona, La Rédaction du journal L'Équipe, op. cit.

31. "L'Équipe, c'est un journal qui parle des stars, pratiquement que des stars. Contrairement à d'autres journaux où il y a des anonymes, il y a des faits divers qui concernent des anonymes, nous non. On ne parle que des stars, donc on sait qu'on vient aussi nous acheter pour ça», explique Gérard Ejnès, le directeur adjoint de la rédaction du quotidien (Arrêt sur images, La Cinquième, 30 mai 1998).

32. Extrait d'une intervention de Christian Quidet lors d'une séance des Lundis de l'INA au Ranelagh (6 juillet 1998) consacrée au «sport à la télévision».

33. Éric Maitrot, Sport et Télé, op. cit., p. 333 et p. 443. 
ne font pas «le poids face à un contrat d'exclusivité» ou vis-à-vis d'un organisateur qui rappelle au journaliste qu'il n'est «que l'employé de son partenaire». Enfin, le choix même des images peut obéir parfois très directement à une logique strictement commerciale, comme dans le cas de certaines compétitions automobiles où des plans sont imposés aux réalisateurs pour des motifs publicitaires.

\section{IMPÉRATIFS EXTERNES ET INTERNES AU CHAMP JOURNALISTIQUE}

Les seules nécessités commerciales ne peuvent expliquer les transformations du mode de traitement de l'information sportive contrairement à ce que pourrait laisser croire la critique politico-éthique sur les «dérives » ${ }^{34}$ du journalisme sportif, très présente chez les journalistes eux-mêmes. Dans une optique sociologique, il apparait plus intéressant de décrire et surtout de comprendre les transformations du traitement de l'information sportive en portant d'abord l'attention sur un premier phénomène qui a été constaté dans d'autres secteurs du journalisme (politique, militaire, social et médical notamment) : la montée de l'expertise est visible à travers les analyses des journalistes et le recours de plus en plus fréquent aux «consultants». L'analyse des commentaires sportifs des grandes rencontres de football à la télévision porte au jour une distinction très forte entre le commentaire sportif traditionnel, qui s'adresse au plus grand nombre, c'est-à-dire qui se veut «populaire», et un commentaire plus technique ou plus spécialisé.

Le premier est incarné par le duo de commentateurs de TF1, Thierry Roland et Jean-Michel Larqué. La nécessité de s'adresser au "grand public », ou plus exactement les représentations que ces deux professionnels se font $\mathrm{du}$ «public» et de ses attentes, se traduisent à travers des catégories de perception très génériques. Il s'agit d'établir une relation complice et rassurante avec les télespectateurs en recourant à des expressions familières et humoristiques ( La défense de..., ce n'est pas la Sécurité sociale ; «Il n'a pas fait le voyage pour rien»; «Ils ne passeront pas leurs vacances ensemble», etc.). Le ton parfois affectif du commentaire vise à montrer la connivence qui règne entre les deux journalistes («Mon cher Jean-Michel», ils font parfois allusion à des paris communs) ou entre confrères (les apartés sur le Variétés club de France, club amateur présidé par Thierry Roland et qui rassemble des journalistes, mais aussi des vedettes du spectacle et d'anciens footballeurs célèbres). Le commentaire peut aussi viser à manifester une forme de proximité avec le «public » (annonce de tournois jeunes, tel ou tel match dédié à des enfants malades ou décédés récemment). Il mobilise de nombreux stéréotypes, notamment nationaux

34. Pour un exemple, $c f$. Jean-François Bourg, «L'information sportive sur un marché monopolistique », Médiaspouvoirs, $\mathrm{n}^{\circ}$ 18, 1990, pp. 29-38. 
(on insiste sur l'impact physique des équipes scandinaves, le fighting spirit des Anglais, les «truquages» des Italiens), et affiche un nationalisme ${ }^{35}$ souvent raillé par les confrères journalistes, sportifs ou non ${ }^{36}$.

À la limite, le spectacle du match est aussi dans le commentaire lui-même, dans les plaisanteries ou les remarques souvent épinglées par deux programmes de Canal+ : le zapping, qui sélectionne quelques moments insolites de la télévision française, ou l'émission «Les Guignols de l'info». Ce qui est perçu très souvent comme des entorses aux règles journalistiques est rendu possible par l'audimat, la popularité de ces deux commentateurs mesurée par les sondages, leur ancienneté37, mais plus largement par les conditions de production de ce commentaire ${ }^{38}$.

Avec l'arrivée de Canal+, dont la diffusion est plus restreinte, s'est développé un autre type de commentaire sportif, qui se veut plus «professionnel» et plus spécialisé. Dans une logique de distinction interne au champ journalistique, Canal+ a non seulement innové dans la mise en images des rencontres de football ${ }^{39}$, et plus largement du sport, mais aussi dans les commentaires. Celui-ci combine à la fois des impératifs commerciaux - en cherchant à s'adresser aux abonnés, qui sont pour une grande part des «connaisseurs» - et des impératifs professionnels, c'est-à-dire une sorte d'art pour l'art du reportage sportif. Si les commentateurs insistent sur l'exclusivité et la qualité du spectacle qui est ou va être offert (voir les bandes annonces ou les titres d'une émission, L'Équipe du dimanche, portant sur les rencontres des championnats étrangers) aux abonnés, s'ils visent à susciter

35. Si le nationalisme est souvent signalé à propos des commentaires sportifs, il est aussi l'une des caractéristiques du traitement des informations à caractère scientifique (par exemple la concurrence entre des équipes françaises et américaines à propos de la recherche contre le sida) ou économique ( $\mathrm{c} f$. certains éditoriaux à propos de la lutte pour la présidence de la future banque centrale européenne) pour ne prendre que ces deux exemples.

36. On pourrait ainsi analyser un supplément de L'Événement du Jeudi (4-10 juin 1998) intitulé «Mondial, le guide anti-beaufs. 32 pages avec tous les bons plans pour fêter la Coupe du Monde sans Thierry Roland».

37. Quand on visionne d'anciens matchs commentés par Thierry Roland au début de sa carrière professionnelle, un des premiers décalages visibles par rapport à ses commentaires contemporains est la sobriété dont il faisait alors preuve.

38. Une analyse sociologique du commentaire sportif à la radio ou à la télévision ne peut, en effet, faire l'économie de ses conditions de production. Cet exercice est très souvent réalisé en direct et sur une durée inhabituelle par rapport aux standards courts de ce média, ce qui implique une tension nerveuse importante. D'autre part, il est très souvent effectué sur le lieu même de la compétition, c'est-à-dire que la passion ou les explosions de joie des commentateurs doivent être rapportées notamment à l'ambiance qui règne sur place.

39. Pour une analyse détaillée de la mise en images de Canal+, $c f$. Francis James, «Le problème de l'évolution du statut de l'image dans l'information télévisée», Bulletin du CERTEIC (Université Lille III), $\mathrm{n}^{\circ}$ 10, 1989, pp. 11-21. 
l'émotion, la passion, ils cherchent aussi à promouvoir un journalisme plus technique, relativement indépendant par rapport aux contraintes commerciales.

Cette double logique, interne et externe, se donne à voir dans la manière de mettre le téléspectateur en position d'être le plus proche possible des «réalités du terrain 40 - c'est-à-dire de la vision des « acteurs » - à travers les précisions données sur des conversations avec les joueurs ou le staff du club, sur les anecdotes récentes qui circulent dans tel ou tel club, sur le déroulement des derniers entraînements et, plus largement, sur la préparation du match. S'appuyant sur des images de vestiaires, d'échanges de regards entre joueurs, sur le suivi des faits et gestes d'un ou plusieurs d'entre eux saisis par des caméras isolées ou encore sur des prises de sons au bord du terrain, les reporters (l'un deux est placé à côté des bancs de touche quand c'est autorisé) permettent de pénétrer dans les coulisses du match de football ${ }^{41}$. Les commentateurs de Canal+ mettent également en exergue une connaissance spécifique du domaine traité avec la lecture de fiches très précises sur les équipes et les joueurs, la mise en place de statistiques individuelles et collectives, des indications sur les tactiques des équipes (ou sur la réalisation de tel ou tel geste) apportées par les consultants de la chaîne, qui sont pour la plupart des anciens footballeurs ou entraîneurs professionnels français.

\section{UNE INFORMATION MOINS STRICTEMENT SPORTIVE}

En plus de la montée de l'expertise, le traitement de l'information sportive a subi une seconde transformation importante. Si elle reste très majoritairement une information de comptes rendus et de résultats sur une «actualité» à la fois programmée (les «calendriers» de la «saison») et, pour une part, imprévisible (les résultats), elle tend à perdre de plus en plus son caractère strictement sportif, c'està-dire qu'elle est traitée sous d'autres aspects : économiques, politiques, médiatiques, scientifiques. Bien que cette évolution doive probablement beaucoup aux transformations déjà évoquées, notamment le poids de plus en plus important des contraintes économiques et les modifications du recrutement des journalistes, elle doit être également liée à la coexistence de définitions concurrentes du métier de journaliste sportif. On peut avancer l'hypothèse qu'il existe une conception

40. Cette intention est réalisée de manière très spectaculaire dans les retransmissions des grands prix de Formule 1, où plusieurs caméras installées dans les voitures des pilotes permettent au téléspectateur de se mettre à la place de celui-ci.

41. Diffusé sur Canal+ en juillet-août 1998, le reportage «Les yeux dans les bleus», qui est le produit du travail d'un journaliste qui a suivi, caméra à la main, l'équipe de France durant toute la Coupe du Monde en France, incarne cette volonté de pénétrer dans la vie quotidienne des grands champions sportifs. 
spécialisée du journalisme sportif. L'information concerne alors l'actualité sportive et strictement l'actualité sportive qui est alors traitée de manière quasi rituelle : donner des résultats, rendre compte des compétitions, des entraînements, recueillir des réactions ${ }^{42}$. Dans certains cas, les journalistes sportifs peuvent se considérer comme des supporters, voire des porte-parole du «mouvement sportif», comme l'illustrent les nombreux ouvrages consacrés aux champions ou à tel ou tel sport (La fabuleuse histoire de...). Dans d'autres, la neutralité journalistique est revendiquée et elle s'exprime, par exemple, dans la mise en exergue des compétences

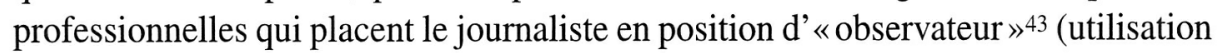
d'outils statistiques).

Une autre conception s'est développée qui se veut plus «généraliste», plus «professionnelle», mettant en avant l'idée que le journalisme sportif est un journalisme comme les autres. Nombre de journalistes sportifs insistent souvent sur le fait «qu'il n'existe pas de journalistes de sport, mais des journalistes tout court» ${ }^{44}$. Les défenseurs de cette conception se veulent souvent plus «critiques", n'hésitant pas à demander un droit de regard sur les affaires sportives (les choix tactiques, les joueurs retenus, le type de recrutement), traitant aussi le sport dans toutes ses dimensions ${ }^{45}$. Par exemple, quand Noël Couedel, directeur de la rédaction à L'Équipe, expliquait en 1988 les «principes» guidant la «politique rédactionnelle» du quotidien, il insistait notamment sur la nécessité de traiter «le sport dans toutes ses dimensions» :

Informer sur le sport de façon classique (résultats, technique, etc.) mais aussi en tenant compte de ses prolongements sociaux, politiques, économiques, culturels. Ne rien occulter des multiples visages du sport moderne, même les plus laids.

C'est surtout chez certains journalistes de la presse écrite (et plus exceptionnellement à la télévision) qu'on retrouve cette conception de l'information. Pour mieux en rendre compte, il faudrait notamment s'interroger sur les trajectoires sociales et professionnelles des «défenseurs» de cette conception : les premiers éléments de notre enquête semblent montrer qu'ils ont souvent fait des études supérieures plus longues que les autres journalistes sportifs ou qu'ils ont (ou ont eu) une

42. Christian Pociello (Les Cultures sportives, Paris, PUF, 1995, pp. 143-145) décrit ainsi ce cycle de l'actualité sportive qui va de la «mise en tension du lectorat» avant la compétition au «retour au calme réflexif et stratégique» sur l'épreuve, après.

43. Francis James, «Mutation des rôles et des images du journaliste de télévision », Médiascope, $\mathrm{n}^{\circ} 1,1992$, pp. 44-48.

44. Eugène Saccomano, chef du service des sports d'Europe 1, cité par Jacques Marchand, $o p$. cit., p. 45. 
activité syndicale et/ou politique. Cette conception de l'information sportive n'est donc aujourd'hui plus seulement présente dans les grands quotidiens et hebdomadaires généralistes mais aussi dans la presse sportive, à commencer par L'Équipe. À travers, par exemple, des dossiers sur le dopage depuis les années Quatre-vingtdix ou sur la commercialisation du sport professionnel, le quotidien spécialisé n’entend plus être considéré comme «la voix du sport français» - il répond ainsi aux reproches fréquents sur la trop grande proximité des journalistes sportifs par rapport à leurs sources d'information - mais travailler «pour les gens qui [1'] achètent », comme l'explique Jérôme Bureau, son rédacteur en chef.

Cette évolution permet de comprendre, au moins en partie, l'un des enjeux les moins visibles de la «polémique » qui a opposé, durant la Coupe du Monde de football en France, des journalistes de la rédaction de L'Équipe, par ailleurs divisée sur le sujet, et Aimé Jacquet, le sélectionneur de l'équipe de France. Si les prises de position dominantes du quotidien étaient ajustées à des intérêts commerciaux, elles s'expliquent aussi en partie par la défense d'une conception plus «critique» du journalisme sportif, notamment à l'égard des dirigeants des grands sports professionnels. Dans ce cas précis, il s'agissait de mettre en cause les choix du sélectionneur, voire ceux de certains (rares) confrères accusés, comme le font remarquer deux journalistes de Libération qui avaient préféré garder une «professionnelle réserve», «de complaisance et de "cocardisme" aigu » ${ }^{46}$. Par exemple, Gérard Ejnès, directeur adjoint de la rédaction de L'Équipe, écrit dans son éditorial du 25 mai 1998 :

Disons-le une fois pour toutes, le rôle de la presse n'est justement pas de se transformer en supporter. Elle est là pour raconter, analyser, expliquer et critiquer si nécessaire. Elle peut se tromper. Qui ne se trompe pas? À elle alors de le reconnaître, de s'incliner devant les faits.

C'est sur les conditions de possibilité du développement de cette conception du journalisme sportif qu'il faut donc s'interroger en comparant les évolutions du sous-champ du journalisme sportif avec d'autres espaces spécialisés du journalisme où une évolution semblable s'est parfois produite ${ }^{47}$. Comme l'ont montré la Coupe

45. On constate que cette lutte pour imposer une conception du journalisme sportif dépasse largement le cas français. $C f$., pour la Belgique, Gérard Derèze, loc. cit., pp. 56-57; pour les ÉtatsUnis, Bruce Garrison avec Mark Sabljak, Sports Reporting, op. cit.

46. Michel Chemin et Christian Losson, « «L'Équipe» à l'heure de l'autocritique», Libération, 14 juill. 1998.

47. Pour le cas du journalisme médical, $c f$. Patrick Champagne avec Dominique Marchetti, "L'information médicale sous contrainte. À propos du "scandale du sang contaminé" ", Actes de la recherche en sciences sociales, 1994, $\mathrm{n}^{\circ}$ 101-102, pp. 40-62. 
du Monde en France et le Tour de France cycliste 1998, le traitement de l'information sportive sous forme d' «affaires» ou de «scandales» tend à se développer, même si c'est dans des proportions beaucoup moins importantes qu'aux États-Unis notamment ${ }^{48}$.

\section{DES CONCEPTIONS DIFFÉRENTES DU JOURNALISME}

Cette opposition sur la manière de pratiquer le journalisme sportif était très présente dans une émission de télévision (Arrêt sur images, La Cinquième, 30 mai 1998) consacrée aux «polémiques» entre certains journalistes et le sélectionneur de l'équipe de France de football, Aimé Jacquet. Tout le débat, présenté par Daniel

\section{JOURNALISME ET COMPÉTENCES (1)}

- Daniel Schneidermann : Est-ce qu'il pourrait vous arriver une fois de le critiquer en face, de lui dire : Aimé là, je pense que vous avez pris une mauvaise décision. Est-ce que ça pourrait vous arriver?

- Thierry Gilardi : Ça pourrait, ça pourrait nous arriver.

- Daniel Schneidermann (ironique) : Ça ne vous est pas encore arrivé.

- Thierry Gilardi : Non, parce que je n'ai pas eu de motif à ça.

- Colombe Schneck : Vous m'avez dit : je ne me sens pas compétent pour juger Aimé Jacquet.

- Thierry Gilardi : Non... oui, il y a un peu de ça.

- Daniel Schneidermann : Ça, c'est une phrase terrible, ça. Si vous n'êtes pas compétent, qui l'est?

- Thierry Gilardi : Je pense que... C'était à propos d'un sujet bien particulier dans le choix de ses joueurs [il évoque l'une des sélections d'Aimé Jacquet]. Pourquoi Dugarry, plutôt qu'Anelka ou Henry? Moi, je répondais, je disais : qui est le mieux placé ? Qui est au centre du groupe? Qui sent les affinités, qui sent les complémentarités? Certainement pas moi. Je vais dormir chez moi. Lui, Jacquet, il va passer deux mois avec tous ces garçons-là.

- Daniel Schneidermann : Non, mais vous vous rendez compte, c'est comme si un journaliste politique disait : oh la la moi, je ne me sens pas compétent pour juger Jospin.

- Thierry Gilardi : [...] Et en plus après, Jospin, on dit quel est le taux de chômage? Quel est le taux d'inflation? Quels sont les résultats du commerce extérieur? On en parlera pendant la Coupe du Monde, pendant la Coupe du Monde. Donnez-nous des matchs, où on jugera après les matchs...

(1) Arrêt sur images, La Cinquième, 30 mai 1998.

48. Pour une analyse de la médiatisation de «scandales sportifs» récents, cf. David Rowe, «Apollo Undone : The Sports Scandal», in James Lull et Stephen Hinerman, Media Scandals, Cambridge, Polity Press, 1997, pp. 203-221. 
Schneidermann, critique de télévision au Monde, et une jeune journaliste de l'émission (Colombe Schneck) était conçu de manière à distinguer en substance le «bon » traitement journalistique de certains organes de la presse écrite (essentiellement L'Équipe), qui avaient osé la critique du sélectionneur, et les journalistes de télévision qui l'avaient ménagé. Ainsi, deux journalistes de télévision, Hervé Mathoux (alors à TF1) et Thierry Gilardi (Canal+), se voyaient reprocher leur traitement trop modéré.

\section{LES MÉDIAS, DES COPRODUCTEURS DES SPECTACLES SPORTIFS}

Au-delà des transformations du territoire journalistique, ce sont aussi les effets que les médias produisent sur les espaces nationaux du football et du basket professionnel qu'il faut saisir. Comme dans bien d'autres domaines (politique, scientifique, etc.), les médias sont devenus, à des degrés divers, un espace stratégique dans la mesure où ils coproduisent les événements de manière de plus en plus directe $^{49}$. Ils contribuent d'abord, pour une large part, à transformer les perceptions publiques du sport-spectacle, que beaucoup de personnes ne connaissent qu'à travers les retransmissions des chaines de télévision. Non seulement, comme le note Charles Suaud, le décalage s'est sans cesse accru entre «le monde social ordinaire» et «l'espace sur lequel les champions [...] sont préparés et entrent en compétition »50 mais les grandes compétitions sportives sont de moins en moins vues sur le lieu même de la compétition au profit de la télévision. Le spectacle sportif est produit deux fois ${ }^{51}$ : d'une part, par les organisateurs et les gestionnaires de la manifestation et, d'autre part, par les professionnels chargés de la production et de la diffusion de la compétition.

Les représentations publiques du sport-spectacle sont donc fortement médiatisées dans tous les sens du terme, les médias construisant un spectacle propre, très différent de celui que les spectateurs peuvent voir sur le terrain. Les chaînes de télévision autonomisent la compétition ${ }^{52}$ en offrant, par le jeu des caméras, des angles différents (ce qui rend par exemple invisible le placement des joueurs ou leur jeu sans ballon), en permettant de revoir certaines actions par le biais du ralenti (qui transforme la vision d'une action vue en direct des tribunes) ou encore en ne livrant que des images très furtives des spectateurs (on ne comprend pas toujours leurs réactions ou leurs calicots). Il y a ainsi peu de domaines où la part du travail de

49. Pour une synthèse des travaux sur le sport-spectacle et les médias, $c f$. Jacques Defrance, Sociologie du sport, Paris, la Découverte (coll. «Repères »), 1995, pp. 62-67.

50. Charles Suaud, «Les états de la passion sportive», Recherches en communication, $n^{\circ} 5,1996$, p. 31.

51. Pierre Bourdieu, «Les Jeux Olympiques. Programme pour une analyse», Actes de la recherche en sciences sociales, $\mathrm{n}^{\circ} 103,1994$, pp. 102-103.

52. Charles Suaud, «Les états de la passion sportive», loc. cit., p. 42. 
construction médiatique est aussi grand. C'est ce spectacle fait par et pour la télévision, qui n'est vu que par le téléspectateur, dont il faut rendre compte. Il s'agit d'analyser les principes de construction du sport-spectacle, c'est-à-dire comment sa production répond aux impératifs (professionnels, économiques, techniques, voire politiques) spécifiques des chaînes. On renverra ici, notamment, aux travaux de Françoise Papa ${ }^{53}$ qui montre les logiques de concurrence ou de division du travail entre médias à l'oeuvre dans les retransmissions télévisées de grands spectacles sportifs.

Le champ journalistique a, en second lieu, contribué à renforcer de plusieurs manières les logiques économiques au sein des espaces nationaux (et plus encore internationaux) du football et du basket ${ }^{54}$. Il suffit notamment de décrire comment l'économie du football professionnel français est aujourd'hui de plus en plus liée à l'économie des médias. Le football français est, d'une certaine manière, dans la même situation que le cinéma, c'est-à-dire que la télévision (et surtout Canal+) est l'un des principaux financeurs de ce spectacle : la part des droits télévisuels dans le budget des clubs est passée de 2 millions en 1980 à 900 millions en 199755 . L'arrivée des télévisions privées a bouleversé l'économie générale du secteur : hausse des salaires, amélioration des installations sportives, arrivée de nouveaux sponsors, importance du merchandising, c'est-à-dire de la vente de produits dérivés, qui dépend entre autres de la visibilité médiatique. À l'inverse, les dirigeants des sports professionnels, en créant des événements qui répondent de plus en plus aux logiques médiatico-économiques, favorisent les audiences de certains supports, ceux-ci étant largement dépendants de ces événements. Pour ne prendre que l'exemple de L'Équipe, ses meilleures ventes sont réalisées au moment des grandes compétitions : Tour de France, finales de Coupe d'Europe avec un club français, Coupe du Monde. Les événements marquants interviennent dans le cas du football en milieu de semaine, et surtout le week-end, d'où l'importance de deux jours : le lundi (480000 exemplaires en moyenne en 1996) et le samedi (420000 exemplaires).

$\mathrm{Si}$ ces interrelations économiques entre les différents espaces des sports professionnels, le champ économique et le champ journalistique ne sont pas

53. Françoise Papa, chercheuse à l'université de Grenoble III, a travaillé sur les retransmissions de plusieurs éditions des Jeux Olympiques sur les chaînes de télévision française.

54. Comme l'écrit Pierre Bourdieu à propos du champ journalistique (Sur la télévision suivi de L'emprise du journalisme, Paris, Liber-Raisons d'agir, 1996, p. 80), «la contrainte structurale que fait peser ce champ, lui-même dominé par les contraintes du marché, modifie plus ou moins les rapports de force à l'intérieur des différents champs, affectant ce que l'on y fait et ce qui s'y produit».

55. Chiffres cités par Patrick Mignon in La Passion du football, Paris, Odile Jacob, 1998, p. 84. 
nouvelles ${ }^{56}$, ni spécifiquement françaises ${ }^{57}$, elles sont aujourd'hui beaucoup plus importantes en raison de l'impact de la diffusion des chaînes de télévision. Les médias français sont, en effet, depuis très longtemps des producteurs directs de spectacles sportifs, qu'ils soient propriétaires d'installations ou surtout organisateurs de compétitions dans le domaine du football, du basket, de l'automobile, du cyclisme. Il suffit de rappeler que c'est L'Équipe (ou plus exactement les éditions Philippe Amaury SA, propriétaires du quotidien) qui organise notamment le Paris-Dakar, le Tour de France, et qui est à l'origine des coupes d'Europe de football et de basket. Mais la nouveauté des années Quatre-vingt et Quatre-vingt-dix tient dans le fait que l'augmentation de la visibilité médiatique du football a favorisé l'arrivée de grandes entreprises, et notamment de grands groupes de communication, dans le football européen qui ont pris des participations dans des grands clubs : Canal+ en France s'est investi dans le Paris-Saint-Germain, Silvio Berlusconi en Italie dans le Milan AC.

Le champ journalistique favorise des logiques économiques concurrentes des logiques sportives, c'est-à-dire internes aux champs relativement autonomes des sports professionnels, en pesant sur l'organisation même des compétitions. Dans le cas du football et du basket, plusieurs compétitions ont été modifiées (et pourraient l'être très prochainement si les grands clubs qui ne sont pas qualifiés sportivement sont «invités» automatiquement à telle ou telle compétition) pour répondre aux impératifs économico-médiatiques. C'est ainsi que les chaînes de télévision contribuent à la fixation du jour (les matchs avancés ou retardés pour une diffusion sur Canal+) et de l'heure des matchs. Il est fréquent que les sportifs (ou les journalistes travaillant pour d'autres médias que la télévision) s'en plaignent, comme ce fut le cas lors de la coupe de la ligue de football 1997/98 : certains matchs télévisés, qui avaient débuté à $21 \mathrm{~h}$, ont fini vers minuit. Les modalités des compétitions, comme l'Euroligue en basket ou la ligue des champions de football, ont été rénovées pour des raisons économiques : augmentation des recettes au guichet, de la publicité mais aussi des rentrées télévisées. Ce qui explique que les matchs à élimination directe ont été supprimés en début de compétition de manière à accroître le nombre de rencontres disputées. De même, ce ne sont plus seulement les clubs champions

56. Pour un exemple, $c f$. un article consacré aux conditions du développement du football américain dans certains pays d'Europe de l'Ouest : Joe Maguire, "The Media-Sport Production Complex : The Case of American Football in Western European Societies », European Journal of Communication, vol. 6, 1991, pp. 315-335. L'auteur montre, à propos de la Grande-Bretagne, les interdépendances économiques entre la National Football League, la chaîne de télévision privée Channel Four et les stratégies marketing de grandes entreprises.

57. Roland Van Gompel, «Sport et médias en Flandre. Caractéristiques et conséquences d'une interdépendance économique », in Gérard Derèze (dir.), La quatrième mi-temps. Contribution à une analyse des relations "sports, médias, société", Université catholique de Louvain-la-Neuve, Observatoire du récit médiatique, 1995, pp. 113-126. 
nationaux qui sont retenus pour disputer ces compétitions mais les seconds, voire les troisièmes (dans le cas du basket) des pays dominants sur le plan sportif, mais aussi de l'audience des chaînes.

Il est beaucoup plus difficile de rendre compte des effets de l'emprise du champ journalistique sur le jeu lui-même ${ }^{58}$. Par exemple, tout donne à penser que la baisse du nombre de buts (et de points dans le cas du basket) marqués ou encore la multiplication des blessures qui est, pour une part, le produit de l'accumulation des matchs dont se plaignent régulièrement les footballeurs n'est pas sans rapport avec les enjeux économiques de plus en plus importants que génère le sport télévisé. Les effets des logiques économico-médiatiques, et notamment l'arrêt Bosman, sont également très importants sur le marché des joueurs - les changements de clubs étant beaucoup plus fréquents qu'au début des années Quatre-vingt - et des entraîneurs, auxquels on demande des résultats immédiats. De même, le champ journalistique, par son travail de construction des «réputations» professionnelles des «équipes» (leurs styles respectifs) ou des sportifs (leur jeu sur le terrain mais aussi leur vie privée) - agit sur les hiérarchies internes de l'espace du football. Comme le résume Thierry Gilardi, journaliste sportif à Canal+, les avis et les notes ${ }^{59}$ de $L ' E ́$ quipe peuvent avoir des effets sur les sportifs eux-mêmes : «Ils [les joueurs de l'équipe de France de football] se souviennent longtemps d'une ligne critique en page 8 de L'Équipe ${ }^{60}$. Les footballeurs ne peuvent, aujourd'hui, plus ignorer complètement les représentations véhiculées par les médias : le fait qu'ils s'en plaignent à l'occasion le démontre. Il faudrait également analyser les effets de la diffusion du football comme spectacle télévisé sur la composition du public présent dans les stades. Nombre de supporters traditionnels, qui sont issus des milieux populaires, tendent - mais des variations importantes peuvent être constatées suivant le type de compétitions et les zones géographiques considérées - à ne plus se rendre au stade en raison, par exemple, de l'augmentation des tarifs, préférant se replier sur les abonnements à des chaînes cryptées et câblées ou sur les retransmissions dans les cafés ou chez des amis

58. On pourrait voir ces effets à propos d'autres sports comme le cyclisme, par exemple. Lors des étapes du Tour de France, on pourrait montrer les effets directs de l'arrivée des caméras de télévision sur la course. En effet, il n'est pas rare qu'elle s'anime soudainement et que des échappées se forment, les professionnels parlant ironiquement d'«échappée publicitaire » pour reprendre une expression employée par Jean-Maurice Ooghe, un des réalisateurs du Tour de France, lors d'une séance des Lundis de l'INA au Ranelagh (6 juill. 1998) consacrée au «sport à la télévision».

59. «Les notes [...] c'est très important. C'est incroyable, mais c'est avec des choses comme ça qu'on fait une carrière », remarque Éric Roy, un joueur de L'Olympique de Marseille (Le Journal du Dimanche, 11 oct. 1998).

60. Propos extraits d'un article de Libération, publié le 31 mai 1998 sous le titre «Double jeu à L'Équipe». 